\title{
A New Multiple Criteria Decision Making Method and its Application in Cloud Computing for Education
}

\author{
http://dx.doi.org/10.3991/ijet.v10i8.5212 \\ Heng Sun \\ Shandong University, Jinan, Shandong, China
}

\begin{abstract}
Cloud computing can extend the traditional education framework. In education, cloud can provide students and teachers with tools to deploy computing resources on-demand for lectures and labs according to their learning needs. But how to select a perfect cloud server is a key point, which is considered as a multiple criteria decision making problem. So, in this paper, intuitionistic fuzzy set is first introduced to express the decision maker's views. Intuitionistic fuzzy set (IFS) includes a membership function and a non-membership function. More importantly, a new operator with choquet integral is developed to deal with assessment of education using cloud computing. Meanwhile, score function and accuracy function are demonstrated to obtain the final result. Finally, we develop this method to apply in a case study to show its applicability.
\end{abstract}

Index Terms-Choquet integral, Cloud computing, Education, Intuitionistic fuzzy set, Multiple criteria decision making.

\section{INTRODUCTION}

In general, cloud computing is a distributed computing paradigm that enables access to virtualized resources including computers, networks, storage, development platform or applications [1-2]. Recently, cloud computing for education has attracted more attention [3-4]. In education, cloud computing caters for desirable properties to provide e-learning services, especially in scenarios where these services are computer-intensive or are offered in a high-scale way. The cloud can provide students and teachers with tools to deploy computing resources ondemand for lectures and labs according to their learning needs [5]. Some educational institutions are already using cloud computing to outsource email services, to offer collaboration tools and data storage for students [6].

The application of cloud-based technologies has been identified as a key trend that enables access to educational services anywhere and promises scalability, enhanced availability and cost savings. Sometimes students require computing power for certain educational applications that are difficult or impossible to run on their own computers with usually limited resources or the institution's servers.

Thus, how to select a perfect cloud server is a key point, which is considered as a multiple criteria decision making problem. The first of step of this problem is to express preferences of the decision maker. But in real world, it is difficult to obtain these preferences. So, intuitionistic fuzzy set is introduced in this paper to express the decision maker's views [7-8].
Atanassov [9] proposed the concept of intuitionistic fuzzy set (IFS) characterized by a membership function and a non-membership function, which is a generalization of the concept of fuzzy set whose basic component of only a membership function [10]. There are more and more researchers focusing on intuitionistic fuzzy set used to deal with multiple criteria decision making problems [11]. Lin [12] presented a new method for handling multiple attribute fuzzy decision making problems, where the characteristics of the alternatives are represented by intuitionistic fuzzy sets. Liu and Wang [13] developed an evaluation function for the decision making problem to measure the degrees to which alternatives satisfy and do not satisfy the decision maker's requirement. In this paper, a new operator with Choquet integral is developed to deal with assessment of education using cloud computing. It is an interesting topic. Choquet integral considers the mutual relationship between different criteria, which are few applied in handling education problems. Finally, we develop this method to apply in a case study to demonstrate its applicability.

The rest of this paper is organized as follows. In Section 2 , we review some concepts of intuitionistic fuzzy sets. Section 3 proposed the new method including intuitionistic fuzzy operator based on Choquet integral, score function and accuracy function. An example is demonstrated in Section 4 to verify the applicability of this method. Finally, Section 5 concludes this paper.

\section{PRELIMINARY}

In this section, some concepts of intuitionistic fuzzy sets are introduced to deal with multiple criteria decision making problem such as assessment of performance of cloud computing in Education.

Definition 1. Let $X$ be a universe of discourse, then a fuzzy set is defined as:

$$
A=\left\{\left\langle x, u_{A}(x)\right\rangle \mid x \in X\right\}
$$

which is characterized by a membership function $u_{A}: X \rightarrow[0,1]$, where $u_{A}$ denotes the degree of membership of the element $x$ to the set $A$.

Definition 2. Let $X$ be an ordinary finite non-empty set. An intuitionisitc fuzzy set in $X$ is an expression $A$ given by

$$
A=\left\{\left\langle x, u_{A}(x), v_{A}(x)\right\rangle \mid x \in X\right\}
$$

where, $u_{A}: X \rightarrow[0,1]$ denotes the degree of membership and $v_{A}: X \rightarrow[0,1]$ denotes the degree of non-membership 
with the condition: $0 \leq u_{A}+v_{A} \leq 1$, for all elements $x$ in the set $X$.

For each intuitionistic fuzzy set $A$ in $X$, if the amount

$$
\pi_{A}(x)=1-u_{A}(x)-v_{A}(x), \forall x \in X .
$$

Then $\pi_{A}(x)$ is called the degree of indeterminacy of the element $x$ to the set $A$. Especially, if

$$
\pi_{A}(x)=1-u_{A}(x)-v_{A}(x)=0, \forall x \in X .
$$

Thus, the intuitionisitc fuzzy set is reduced to a fuzzy set. For computational convenience, in this paper, $\left(u_{A}(x)\right.$, $\left.v_{A}(x)\right)$ is called as an intuitionistic fuzzy number.

Definition 3[14] Let $a=\left(u_{a}, v_{a}\right)$ and $b=\left(u_{b}, v_{b}\right)$ be two intuitionistic fuzzy numbers, then it can be obtained that

(1) $a \oplus b=\left(u_{a}+u_{b}-u_{a} u_{b}, v_{a} v_{b}\right)$;

(2) $a \otimes b=\left(u_{a} u_{b}, v_{a}+v_{b}-v_{a} v_{b}\right)$;

(3) $\lambda a=\left(1-\left(1-u_{a}\right)^{\lambda},\left(v_{a}\right)^{\lambda}\right), \lambda>0$;

(4) $a^{\lambda}=\left(\left(u_{a}\right)^{\lambda}, 1-\left(1-v_{a}\right)^{\lambda}\right), \lambda>0$.

Definition 4[15] Given two intuitionistic fuzzy values $A$ and $B$, the following operations are valid:

(1) $a \oplus b=b \oplus a$;

(2) $\lambda_{1}(a \oplus b)=\lambda_{1} a \oplus \lambda_{1} b$;

(3) $\lambda_{1} a \oplus \lambda_{2} a=\left(\lambda_{1}+\lambda_{2}\right) a$.

\section{PROPOSED METHOD WITH INTUITIONISTIC FUZZY} SET

In this section, to evaluate the performance of cloud computing in education, a new proposed method is introduced.

\section{A. Description of the problem}

For an evaluation problem, we construct the decision matrix $D=\left[\&_{i}\right]_{m \times n}$, where all the arguments $\alpha_{\varphi}(i=1,2, \ldots m ; j=1,2, \ldots n)$ are intuitionistic fuzzy numbers provided by the decision maker. As for every alternative $a_{i}(i=1,2, \ldots, m)$, the decision maker is invited to express evaluation or preference according to each criterion $c_{j}(j=1,2, \ldots, n)$ by a intuitionistic fuzzy element $\&_{i j}=\left(u_{i j}, v_{i j}\right)(i=1,2 \mathrm{~L}, m ; j=1,2, \mathrm{~L}, n)$, where $u_{i j}$ indicates the hesitant degree that the decision maker considers what the alternative $\alpha_{i}$ should satisfy the criteria $c_{j}, v_{i j}$ indicates the hesitant degree that expert e considers what the alternative $\alpha_{i}$ should not satisfy the criteria $c_{j}$. Then we can obtain a decision making matrix as follow:

$$
D=\left(\begin{array}{cccc}
\tilde{a}_{11} & \tilde{a}_{12} & \cdots & \tilde{a}_{1 n} \\
\tilde{a}_{21} & \tilde{a}_{22} & \cdots & \tilde{a}_{2 n} \\
\vdots & \vdots & \ddots & \vdots \\
\tilde{a}_{m 1} & \cdots & \cdots & \tilde{a}_{m n}
\end{array}\right)
$$

\section{B. Intuitionistic fuzzy operators and value function}

For multiple criteria decision making problems, it does not need assumption that criteria or preferences are independent of one another, and was used as a powerful tool for modeling interaction phenomena in decision making. As an aggregation operator, the Choquet integral has been proposed by many researchers as an adequate substitute to the weighted arithmetic mean or OWA operator to aggregate criteria [16-18].

Definition 5. A fuzzy measure on $X$ is a set function $u$ : $P(X) \rightarrow[0,1]$, satisfying the following conditions:

(1) Boundary condition: $u(\Phi)=0, u(X)=1$;

(2) Monotonicity: if $A, B \in P(X)$ and $A \subseteq B$ then $u(A) \leq$ $u(B)$.

Here, $u(A)$ can be regarded as the grade of subjective importance of decision criteria set $A$. The similar situation is to $u(B)$. Then, we could obtain the following operations.

(1) if $u(A \mathrm{U} B)=u(A)+u(B)$, then $A$ and $B$ are considered to be without interaction (or to be independent), which is called an additive measure.

(2) if $u(A \mathrm{U} B)>u(A)+u(B)$, then $A$ and $B$ are considered to be a positive synergetic interaction (or complementary), which is called a super-additive measure.

(3) if $u(A \mathrm{U} B)<u(A)+u(B)$, then $A$ and $B$ are considered to be a negative synergetic interaction (or substitutive), which is called a sub-additive measure.

Definition 6. Let $f$ be a positive real-valued function on $X$. The discrete Choquet integral of $f$ with respect to $u$ is defined by

$$
C(f)=\sum_{i=1}^{n} f_{(i)}\left[u\left(A_{(i)}\right)-u\left(A_{(i+1)}\right)\right]
$$

where (.) denotes a permutation on $X$ such that $f_{(1)} \leq f_{(2)}$ $\leq \ldots \leq f_{(n)}$. Also $A_{(i)}=\{1, \ldots, n\}, A_{(n+1)}=\Phi$. Obviously, this Choquet integral is a linear expression up to a reordering of the elements.

Definition 7. Let $a_{i}=\left(u_{i}, v_{i}\right)(i=1,2, \ldots, n)$ be a collection of intuitionistic fuzzy numbers on $X$, and $u$ be a fuzzy measure on $X$. The intuitionistic fuzzy operator based on Choquet integral of $a_{i}$ with respect to $u$ is defined by

$$
\begin{aligned}
& I F\left(a_{1}, a_{2}, \ldots, a_{n}\right)=a_{(1)}\left(u\left(A_{(1)}\right)-u\left(A_{(2)}\right)\right) \oplus a_{(2)}\left(u\left(A_{(2)}\right)-u\left(A_{(3)}\right)\right) \\
& \oplus \ldots \oplus a_{(n)}\left(u\left(A_{(n)}\right)-u\left(A_{(n+1)}\right)\right) \\
& =\sum_{i=1}^{n}{ }^{\oplus} a_{(i)}\left(u\left(A_{(i)}\right)-u\left(A_{(i+1)}\right)\right)
\end{aligned}
$$

(4)

where (.) denotes a permutation on $X$ such that $a_{(1)} \leq a_{(2)}$ $\leq \ldots \leq a_{(n)}$. Also $A_{(i)}=\{1, \ldots, n\}, A_{(n+1)}=\Phi$.

Definition 8. Let $a_{i}=\left(u_{i}, v_{i}\right)(i=1,2, \ldots, n)$ be a collection of intuitionistic fuzzy numbers on $X$, and $u$ be a fuzzy measure on $X$. If all $a_{i}(i=1,2, \ldots, n)$ are equal, that is, for all $i, a_{i}=a=\left(u_{a_{i}}, v_{a_{i}}\right)$, then $\operatorname{IF}\left(a_{1}, a_{2}, \ldots, a_{n}\right)=a$.

Definition 9. Let $a_{i}=\left(u_{a_{i}}, v_{a_{i}}\right)(i=1,2, \ldots, n)$ and $b_{i}=\left(u_{b_{i}}, v_{b_{i}}\right)(i=1,2, \ldots, n)$ be two collections of 
intuitionistic fuzzy numbers on $X$, and $u$ be a fuzzy measure on $X$. (.) denotes a permutation on $X$ such that $a_{(1)}$ $\leq a_{(2)} \leq \ldots \leq a_{(n)}$ and $b_{(1)} \leq b_{(2)} \leq \ldots \leq b_{(n)}$. If $a_{(i)} \leq b_{(i)}$ for all $i$, that is, $u_{a_{i}}<u_{b_{i}}$ and $v_{a_{i}}>v_{b_{i}}$, then

$$
\operatorname{IF}\left(a_{1}, a_{2}, \ldots, a_{n}\right) \leq \operatorname{IF}\left(b_{1}, b_{2}, \ldots, b_{n}\right)
$$

Definition 10. Let $a=(u, v)$ be an intuitionsitic fuzzy number, a score function $S$ of an intuitionistic fuzzy number can be represented as follows:

$$
S(\alpha)=u-v, S(\alpha) \in[-1,1]
$$

Definition 11. Let $a=(u, v)$ be an intuitionsitic fuzzy number, a accuracy function $H$ of an intuitionistic fuzzy number can be represented as follows:

$$
H(\alpha)=u+v, H(\alpha) \in[0,1]
$$

The larger the value of $H(\alpha)$ is, the more the degree of accuracy of the intuitionistic fuzzy number is.

Hong and Choi [19] represented that the relation between the score function $S$ and the accuracy function $H$ is similar to the relation between mean and variance in statistics. According to the score function $S$ and the accuracy function $H, \mathrm{Xu}$ [20] proposed an order relation between two intuitionistic fuzzy numbers to further compare these two intuitionistic fuzzy numbers in the following:

Definition 12. Let $a_{1}=\left(u_{1}, v_{1}\right)$ and $a_{2}=\left(u_{2}, v_{2}\right)$ be two intuitionistic fuzzy numbers, $S\left(a_{1}\right)=u_{1}-v_{1}$ and $S\left(a_{2}\right)=u_{2}$ $v_{2}$ be the scores of $a_{1}$ and $a_{2}$, respectively, and let $H\left(a_{1}\right)=$ $u_{1}+v_{1}$ and $H\left(a_{2}\right)=u_{2}+v_{2}$ be the accuracy degrees of $a_{1}$ and $a_{2}$, respectively, then we can acquire the following characteristics:

(1) if $S(a)<S(b)$, then the number $a$ is smaller than $b$, denoted by $\mathrm{a}<\mathrm{b}$;

(2) if $S(a)=S(b)$, then the accuracy function should be introduced to compare two intuitionistic fuzzy numbers. Thus, we can obtain that

1) if $H(a)<H(b)$, then the number $a$ is smaller than $b$, denoted by $a<b$;

2) if $H(a)<H(b)$, then the number $a$ is equal to $b$, denoted by $a=b$.

The operational laws of $\mathrm{S}$ and $\mathrm{H}$ can be applied in the process of aggregation of information in decision matrix.

\section{Process of proposed method}

In this section, we will propose a procedure to form this evaluation model based on mentioned methods, where values take the form of intuitionistic fuzzy numbers. The procedure includes the following steps:

Step1. Based on the problem background, a decision maker is invited to provide several attributes and alternatives.

Step 2. The decision maker constructs a decision matrix based on his/her preferences.

Step 3. The attribute weights of the $n$ attributes denoted as $w=\left(w_{1}, w_{2}, \ldots, w_{n}\right)^{T}$ with $0 \leq w_{j} \leq 1(j=1,2, \ldots, n)$ and $\sum_{i=1}^{n} w_{j}=1$ can be determined.

Step 4. Using discrete Choquet integral, he intuitionistic fuzzy operator based on Choquet integral can be introduced to aggregate information of each alternative on each attribute.

Step 5. The score function and the accuracy function is defined to compare different intuitionistic fuzzy numbers related to alternatives.

Step 6. The rank-order can be obtained. Then, we can select optimal alternative by the largest score.

Step 7. End.

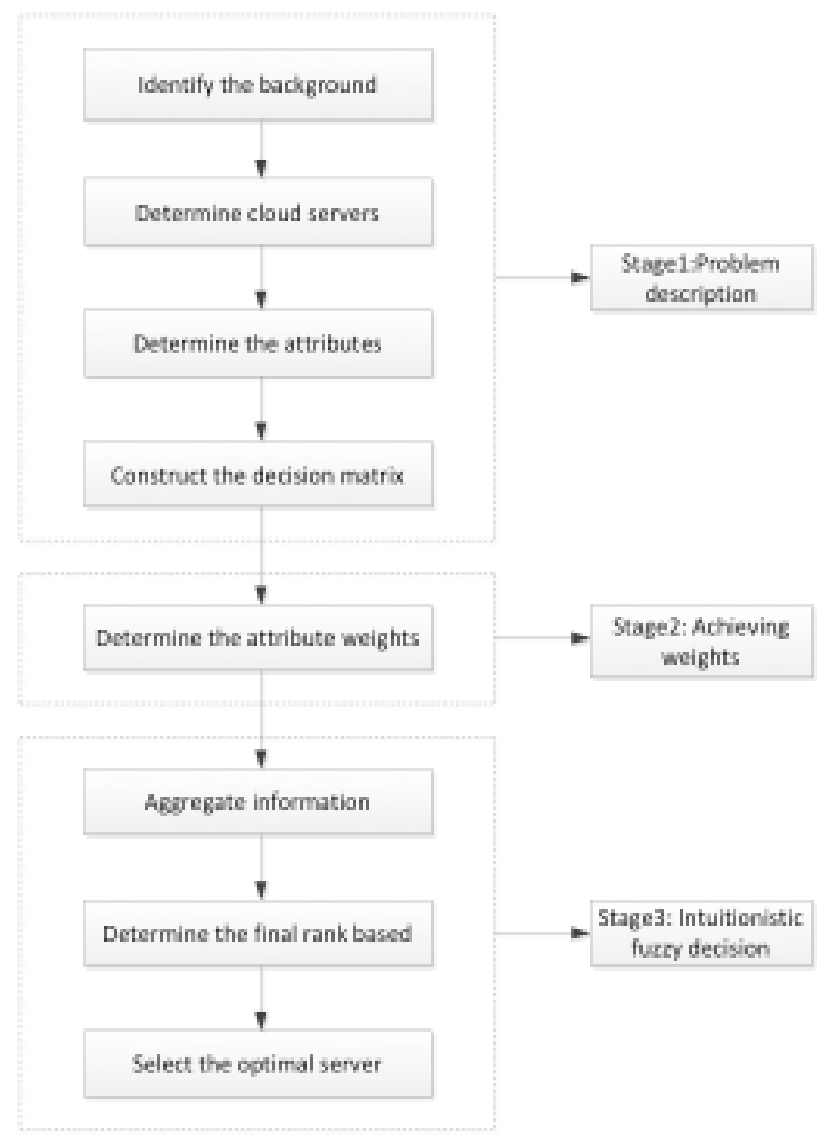

Figure 1. The process of the proposed method

\section{ILLUSTRATIVE EXAMPLE}

Based on the proposed framework in Figure 1, we invite a famous expert as the decision maker to implement process of decision making. Three companies which provide cloud service for educational system are selected by the decision maker as the alternatives including $A_{1}, A_{2}$, $A_{3}$ and $A_{4}$. Then, the decision maker identifies three criteria denoted as quality $C_{1}$, price $C_{2}$, and consumer satisfaction $C_{3}$. Then, the decision maker gives preferences of each alternative on each criterion, respectively. Therefore, the intuitionistic fuzzy decision matrix $D=\left[a_{i j}\right]_{4 ¥ 3}$ can be illustrated in Table 1 .

TABLE I.

ORIGINAL INTUITIONISTIC FUZZY DECISION MATRIX

\begin{tabular}{|c|c|c|c|}
\hline & $C_{1}$ & $C_{2}$ & $C_{3}$ \\
\hline$A_{1}$ & $\{0.2,0.5,0.3\}$ & $\{0.6,0.3,0.1\}$ & $\{0.3,0.5,0.2\}$ \\
\hline$A_{2}$ & $\{0.4,0.5,0.1\}$ & $\{0.2,0.5,0.3\}$ & $\{0.3,0.5,0.2\}$ \\
\hline$A_{3}$ & $\{0.3,0.3,0.4\}$ & $\{0.4,0.4,0.2\}$ & $\{0.4,0.5,0.1\}$ \\
\hline$A_{4}$ & $\{0.7,0.2,0.1\}$ & $\{0.5,0.5\}$ & $\{0.8,0.1,0.1\}$ \\
\hline
\end{tabular}


After obtaining the decision matrix, the decision maker provides the fuzzy measure of these three criteria denoted as $\lambda=(0.3,0.3,0.4)^{T}$. Then, the intuitionistic fuzzy operator based on Choquet integral from Eq. (4) is applied to aggregation preferences of the decision maker in Table1.

TABLE II.

THE RANKING ORDER

\begin{tabular}{|c|c|c|}
\hline & score & rank \\
\hline$A_{1}$ & 0.36 & 3 \\
\hline$A_{2}$ & 0.25 & 4 \\
\hline$A_{3}$ & 0.38 & 2 \\
\hline$A_{4}$ & 0.46 & 1 \\
\hline
\end{tabular}

Based on the Definition 10 and Definition 11, it can be calculated the score and accuracy of each alternative shown in Table II. Finally, using the operational laws related to score function and accuracy function in Definition 13, the ranking order of alternatives can be acquired in Table II which is demonstrated as $A_{4} \mathrm{f} \quad A_{3}$ f $A_{1} \mathrm{f} \quad A_{2}$. From this ranking order, we can select $A_{4}$ as the best choice.

\section{CONCLUSIONS}

As we know, cloud computing is a distributed computing paradigm that enables access to virtualized resources including computers, networks, storage, development platform or applications. More importantly, cloud computing can extend the traditional education framework. In education, cloud can provide students and teachers with tools to deploy computing resources ondemand for lectures and labs according to their learning needs. But how to select a perfect cloud server is a key point, which is considered as a multiple criteria decision making problem. So, in this paper, intuitionistic fuzzy set is first introduced in this paper to express the decision maker's views. Intuitionistic fuzzy set (IFS) includes a membership function and a non-membership function A new operator with Choquet integral is developed to deal with assessment of education using cloud computing. Finally, we develop this method to apply in a case study to demonstrate its applicability. Although the proposed method in this paper can deal with assessment of cloud server, it cannot address more complex problems such as group decision making. Then, we will extend this method in group decision making in the future.

\section{REFERENCES}

[1] J. A. Gonzalez-Martinez, M.L. Bote-Lorenzo, E. Gomez-Sanchez, R. Cano-Parra. Cloud computing and education: A state-of-the-artsurvey, Computers \& Education, $80 \quad$ (2015) 132-151. http://dx.doi.org/10.1016/j.compedu.2014.08.017

[2] N. M. Abrams, Combining cloud networks and course management systems for enhanced analysis in teaching laboratories, Journal of chemical education, 89(4) (2012) 482-486. http://dx.doi.org/10.1021/ed200160d

[3] E. AbuKhousa, N. Mohamed, J. Al-Jaroodi,. e-Health cloud: opportunities and challenges. Future Internet, 4(3) (2012) 621-645. http://dx.doi.org/10.3390/fi4030621

[4] A. Belahcen, M. Abik, R. Ajhoun, C-MADAR Learning: cloud based learning environment, In Proceedings of the 2012 IEEE Global Engineering Education Conference (EDUCON '12) (2012) (pp. 1e7). http://dx.doi.org/10.1109/EDUCON.2012.6201064
[5] L. Johnson, S. Adams, M. Cummins, NMC horizon report: 2012 higher education edition, Technical Report. The New Media Consortium (2012).

[6] A.F. Hegazy, A.E. Khedr, Y.A. Geddawy, An adaptive framework for applying cloud computing in virtual learning environment at education a case study of "AASTMT", Procedia Computer Science, 65 (2015) 450-458. http://dx.doi.org/10.1016/j.procs.2015.09.121

[7] T.Y. Chen, A comparative analysis of score functions for multiple criteria decision making in intuitionistic fuzzy settings, Information Sciences, $181 \quad$ (2011) 3652-3676. http://dx.doi.org/10.1016/j.ins.2011.04.030

[8] K.T. Atanassov, Intuitionistic fuzzy sets, in: Seventh Scientific Session of ITKR, Sofia, 1983.

[9] K.T. Atanassov, Intuitionistic fuzzy sets, Fuzzy sets and systems, 20(1) (1986) 87-96. http://dx.doi.org/10.1016/S0165-0114(86) 80034-3

[10] A. Pankowska, M. Wygralak, General IF-sets with triangular norms and their applications to group decision making. Information Sciences, $176 \quad$ (2006) 2713-2754. http://dx.doi.org/10.1016/j.ins.2005.11.011

[11] G.W. Wei, Some induced geometric aggregation operators with intuitionistic fuzzy information and their application to group decision making, Applied Soft Computing, 10 (2010) 423-431. http://dx.doi.org/10.1016/j.asoc.2009.08.009

[12] L. Lin, X. H. Yuan, Z.Q. Xia, Multicriteria fuzzy decision-making methods based on intuitionistic fuzzy sets. Journal of Computer and System Sciences, 73 (2007) 84-88. http://dx.doi.org/10.1016/ j.jcss.2006.03.004

[13] H.W. Liu, G.J. Wang, Multi-criteria decision-making methods based on intuitionistic fuzzy sets. European Journal of Operational Research, 179 (2007) 220-233. http://dx.doi.org/10.1016/j.ejor. 2006.04.009

[14] S.M. Chen, T.S. Li, Evaluating students' answerscripts based on interval-valued intuitionistic fuzzy sets, Information Sciences, 235 (2013) 309-322. http://dx.doi.org/10.1016/j.ins.2012.12.031

[15] Z.S. Xu, A method based on distance measure for interval-valued intuitionistic fuzzy group decision making, Information Sciences 180 (1) (2010) 181-190. http://dx.doi.org/10.1016/j.ins. 2009.09.005

[16] C.Q. Tan, X.H. Chen, Intuitionisic fuzzy Choquet integral operator for multi-criteria decision making, Expert Systems with Applications, 37 (2010) 149-157. http://dx.doi.org/10.1016/j.eswa. 2009.05.005

[17] C. Labreuche, M. Grabisch, The Choquet integral for the aggregation of interval scales in multicriteria decision making, Fuzzy Sets and Systems, 137 (2003) 11-26. http://dx.doi.org/10.1016/S0165-0114(02)00429-3

[18] J.D. Qin, X.W. Liu, Study on interval intuitionistic fuzzy multiattribute group decision making method based on Choquet integral, Information Technology and Quantitative Management, ITQM 17 (2013) 465-472. http://dx.doi.org/10.1016/j.procs.2013.05.060

[19] D.H. Hong, C.H. Choi, Multicriteria fuzzy problems based on vague set theory, Fuzzy Sets and Systems 114 (2000) 103-113. http://dx.doi.org/10.1016/S0165-0114(98)00271-1

[20] Z.S. $\mathrm{Xu}$, Intuitionistic fuzzy aggregation operators. IEEE Transaction on Fuzzy Systems, 15 (2007) 1179-1187. http://dx.doi.org/10.1109/TFUZZ.2006.890678

\section{AUTHOR}

Heng Sun is with the Modern Educational Technology Lab School of Foreign Languages, Shandong University, Jinan, Shandong, China. His research directions are computer technology application, network information technology and modern education informatization. (email:1263307508@qq.com).

This work was supported in part by Science and Technology Planning Project of Shandong Province, China (Year: 2014; No: 2014GGX101018). Submitted 07 November 2015. Published as resubmitted by the author 05 December 2015. 